\title{
COMMISSION 42: CLOSE BINARY STARS (ÉTOILES BINAIRES SERRÉES)
}

\author{
Report of Meetings, 22, 23, 28 and 29 August 1973
}

President: M. Plavec.

SECRETARY: I. Jurkevich.

\section{Business Meetings}

The business meetings were held on August 22 and 28, and this summary covers both of them. A. H. Batten remembered briefly the deceased IAU members O. Günther, A. H. Joy and H. Shapley. Draft Reports could not be discussed since to most members they had not been accessible. The President promised to distribute the reprints to all members as soon as the reprints are available. The Commission was very strongly in favor of continuing the style and publication of the Reports on Astronomy in spite of the high price of the volume. It is recommended to future Presidents to consider the possibility of distributing a mimeographed form of the Commission report to members before the General Assembly.

The name of the Commission was discussed, also with other Commissions (26 and 30). It was decided to change the name to Close Binary Stars, thereby indicating that the field of the Commission will from now on include eclipsing binaries, spectroscopic binaries, and all other types of binary stars that are close enough in space to invoke serious interaction between the components. Visual double stars as well as astrometric binaries continue to be the domain of Commission 26, as before.

The Organizing Committee presented the following slate for election: President: T. Herczeg, Vice-President: G. Larsson-Leander, Organizing Committee: A. H. Batten, M. Fracastoro, K. Gyldenkerne, M. Kitamura, D. M. Popper, J. Smak, F. B. Wood, M. Plavec (Past President). $\mathrm{K}$. O. Wright proposed to add two more names to improve the geographical distribution and increase the representation of young astronomers. The Organizing Committee then proposed to add E. P. J. van den Heuvel and B. Warner, and in this form the list of new officers was approved by the Commission.

The Commission elected 18 new members, partly from young astronomers who just became members of the IAU, partly from among astronomers working in the broader field covered by the new name of the Commission. Future procedures were discussed for election or co-option of new members. It was decided unanimously that if a National Committee proposes a new Commission member, this proposal will be considered only provided satisfactory documentation is submitted.

The by-laws of the Commission were discussed. It was agreed that as a rule a member of the Organizing Committee should not serve for more than three terms unless he becomes Vice-President or President. The President is expected to serve one term after his office term has expired.

The Commission acknowledged the work done by Larsson-Leander and his staff together with the regional contributors on compiling and disseminating the Bibliography on Eclipsing Variables. Members consider this to be a valuable publication; Larsson-Leander reported that in fact the circulation is wider, 200 copies being distributed. He promised to continue the semiannual publication of the Bibliography for the next three years. The Commission then voted to ask the Executive Committee to grant a subsidy of $\$ 200$ to defray partly the costs. A vote of thanks was also expressed to Drs Detre and Szeidel of the Budapest Observatory for the excellent services rendered to the Commission members by the frequent and rapid publication of the Information Bulletin on Variable Stars.

A. H. Batten then raised the question of need for the 7th Catalog of Orbital Elements of Spectroscopic Binary Systems, since the 6th Catalog will soon not be available. The Commission feels that the 7 th Catalog will soon be needed and strongly recommends its preparation. 
A Symposium on Be Stars and Shell Stars will be organized by Commissions 42, 29 and 36, probably in September 1975. $M$. Plavec will represent Commission 42 on the Organizing Committee. Co-sponsoring a joint Colloquium on Variable Stars in Budapest in September 1975 was also discussed, but further discussion on the program appears necessary.

$Y$ Kondo presented a brief report of the sub-committee on extraterrestrial observations. Members of this sub-committee are expected to work as local disseminators of information. $K$. Gyldenkerne discussed the problems of coordinated programs; these will be continued but no new stars have been added this time. A. H. Batten announced that the working group on Spectroscopic Binary Stars of Commissions 30 and 42 has now in fact been transferred to Commission 42 .

The Commission discussed in detail the priorities for research in the field of the Commission. The Organizing Committee joined by M. V Ovenden then prepared the following document, which was approved by the Commission.

\section{Priorities for Research}

Within the broad spectrum of the physical sciences, astrophysics is important as it studies matter under a variety of physical conditions not accessible in the laboratory. In the study of close binary stars, we approach most closely to laboratory experimentation since we examine the effects of one star upon another under a great variety of conditions.

When the stars are sufficiently close together to form an eclipsing pair, but sufficiently detached not to affect their structure significantly, such a non-interacting binary star provides the best available data on stellar masses, radii, luminosities, and other characteristics. When, on the other hand, the stars do interact strongly, their evolution will be completely different from the evolution of a single star. Since a large fraction of stars actually belong to binary or multiple systems, the study of the group of interacting binary systems is essential for our understanding of stars as a whole.

The non-interacting and interacting binary systems thus represent two different but closely related approaches to further research on close binary systems, each of which must be treated separately. The following projects and problems are considered to be of top priority in the near future. (The order in which they are listed does not imply relative importance.)

\section{A. NON-INTERACTING CLOSE BINARY SYSTEMS}

(1) Obtaining reliable masses of representative types of stars. While every mass determination, if reliable, is of importance, priority should be given to investigating early-type stars and stars evolved away from the main sequence. Infrared spectroscopy is important in this case.

(2) More emphasis should in general be placed on photometric and spectroscopic observations of eclipsing and spectroscopic binary systems of moderate and longer periods, since they are important for evolutionary studies and they provide data on evolved stars.

(3) A new and very promising method of obtaining dimensions of stars and binary systems is the Narrabri Intensity Interferometer. The successful studies of Alpha Virginis and Gamma Velorum convince us that a larger instrument of the same type would be of great value to the progress in studying not only single stars but binary systems as well.

(4) Since binary stars form a significant portion of the whole stellar population, and since their evolution strongly depends on masses and dimensions of the systems, it is essential to rediscuss the frequency distribution of binary stars with luminosity and spectral type. A new classification scheme is needed, in particular for eclipsing binaries.

\section{B. INTERACTING CLOSE BINARY SYSTEMS}

(1) Concentrated effort should be made to detect compact and collapsed objects in binary systems. Systems suspected of containing a black hole, such as Cyg X-1, Beta Lyrae, Epsilon Aurigae and others, should be studied by all available methods. Since the classical spectral observations in the 
visible range have not given sufficient information, of particular importance become the observations from artificial satellites (far UV and X-rays), observations in the infrared, and radio observations.

(2) The existence of compact binary sources of X-rays emphasizes once more the necessity of investigating mass transfer in close binary systems, in particular the interaction of the gas streams with the mass-accreting component. Change of structure and evolution of this star as well as formation of disks and extended envelopes around it may explain a wealth of other phenomena, for example, the shell stars and many peculiar objects like novae, U Gem stars, symbiotic objects, etc.

(3) The studies mentioned sub $B(1)$ and $B(2)$ represent applications, of particular current importance, of general investigations on evolution of close binaries accompanied by mass exchange and mass loss. It has become clear that the results of such investigations apply to a wide variety of systems in which a great and unexpected range of physical phenomena occur. Hence it is necessary that investigations of the evolution of interacting systems be applied to an even wider range of conditions than before.

(4) Associated with the preceding problems are further theoretical topics such as hydrodynamical treatment of gas flow, time scales for synchronization of rotation of binary star components, physics of and radiation transfer in extended atmospheres and circumstellar disks. Resulting models should be compared with new spectrsocopic observations.

(5) The observed radio emission from several binary systems still represents a complete puzzle. More observations on radio frequencies as well as complex observational and theoretical studies of radio binaries are necessary.

(6) For the elucidation of many aspects of the physics of close binary systems, it is important that observations of various kinds be repeated on a number of selected systems over a considerable interval of time in order that the time-varying phenomena may be studied.

\section{Scientific Meetings}

Meeting of August 29, 1973, on Current Trends in Binary Star Studies. Eight short reports were presented as follows: K. O. Wright on spectral studies of VV Cephei; R. E. Wilson on his program computing photometric elements from light curves; J. B. Hutchings on the problem of reconciling mass ratios obtained photometrically and spectroscopically; D. M. Popper surveyed his work on deriving masses of detached systems, Algol systems, and binaries with $\mathbf{H}$ and $\mathrm{K}$ line emission; M. G. Fracastoro suggested that a search be made for new eclipsing binaries in the equatorial belt, within the magnitudes $7^{m}$ to $9^{m}$ Y. Kondo reported on his observations of the He I line at $\lambda 10830$, and on his and McCluskey's analysis of LY Aur. F. B. Wood reported on the observations of $\gamma^{2}$ Velorum, which show fairly rapid variability in He II. No definite eclipses were detected. M. Plavec announced results of theoretical computations of mass loss from red giants with deep convective envelopes.

Joint meeting with Commissions 27, 40, and 44 on X-Ray Sources, Radio Sources and Black Holes in Binary Systems. It was held on August 23 and is reported by Commission 27. Contributions by members of Commission 42 included a new model of $\beta$ Lyrae (R. E. Wilson) and a discussion of mass determination in X-ray binaries ( $\mathrm{Y}$. Kondo). 